\title{
Lipschutz Ulcer in A Virgin Woman: A Case Report
}

\author{
I Gusti Ayu Agung Elis Indira, Vanessa Vijayamurthy, Made Dwi Puspawati, Anak \\ Agung Gde Putra Wiraguna \\ Department of Dermatology and Venereology, Faculty of Medicine Universitas Udayana/Sanglah \\ General Hospital, Bali, Indonesia
}

\begin{abstract}
Background: Lipschutz ulcer, also known as vulvae acutum ulcer, is an acute ulcer in the vulva. The aetiology and pathogenesis of Lipschutz ulcer are unclear, but it is known to be associated with infectious diseases such as Mycoplasma infection, paratyphoid fever, influenza A, and most infections with Epstein-Barr virus (EBV). This ulcer appears in adolescent females aged 14-20 years old, with 70\% of cases occurring in virgin women. Purpose: To review the causes of non-Sexually Transmitted Diseases (STD) ulcers, so clinicians can establish an accurate diagnosis and rational therapy; therefore, minimizing the psychological impact on the patient due to possible misdiagnosis of STD-induced ulcer. Case: A painful wound in the genital area of a 20-year-old virgin woman. She was diagnosed with Lipshutz ulcer and vulvovaginal candidiasis based on the anamnesis, physical examination, and laboratory examination obtained from vaginal discharge using potassium hydroxide, Gram staining, and blood test to exclude genital ulcer caused by the sexually transmitted agent. A blood test was taken, including the serological tests for syphilis and genital herpes. Tests for EBV were also performed. The patient was treated only with single-dose fluconazole $150 \mathrm{mg}$ orally and saline compress on the ulcer. Genital ulcer and vaginal discharge improved one week after treatment. Discussion: Lipshutz ulcer management is symptomatic, usually self-limiting, and disappears spontaneously within 1-2 weeks without recurrences. It can also occur as a single lesion with possible coinfection of other agents, for example, candidiasis, which in this case, requires treatment of comorbidities. Hygiene factors play an essential role in preventing occurrence of the secondary infection and further development of the disease. Conclusion: The diagnosis of Lipshutz ulcer is mainly based on clinical manifestation after excluding several possible ulcers that can affect the genital area, including sexually transmitted infections.
\end{abstract}

Keywords: Lipschutz ulcer, vulvovaginal candidiasis, self limiting disease.

Correspondence: I Gusti Ayu Agung Elis Indira. Department of Dermatology and Venereology, Faculty of Medicine Universitas Udayana/Sanglah General Hospital, Jl. Diponegoro, Dauh Puri Klod Denpasar 80113. Indonesia. Phone: +62361 227912, email: elisindira@yahoo.com.

\section{BACKGROUND}

Lipschutz Ulcers (LU) or vulvae acutum ulcers are an acute ulcer on the vulva. It has characteristic forms of vulva edema and necrotic ulcer pain with rapid onset. $\mathrm{LU}$ is a rare condition. To date, the incidence of LU has been undocumented, and only a few case reports and case series have been found. However, there has been an incidence of ulcus vulvae acutum related to Epstein-Barr virus (EBV) on 10 $30 \%$ of acute genital ulcers in adolescent women. It is common in women who are not sexually active or virgin. These ulcers occur in adolescent women aged 14-20 years old, and 70\% of cases occurred in virgin women. $1,2,3,4$

The pathogenesis of LU is unknown, but it is associated with infectious diseases such as Mycoplasma infection, paratyphoid fever, influenza A infection, and mostly with EBV infection., ${ }^{2,5,6}$ Diagnosis is mainly based on clinical grounds after excluding some possible ulcers that may affect the genital area. LU management uses symptomatic therapy and mostly self-limited. LU disappears spontaneously within two weeks without recurrence. ${ }^{2}$

Sexually transmitted infections (STI) often cause ulcers that affect the genital area. However, the cause of non-STI should be considered. Understanding these diseases helps in establishing an accurate diagnosis and therapy and minimizing the psychological impact on the patient due to misdiagnosis of STD-induced ulcers. This case was reported to raise an understanding of the aetiology, clinical, investigation, and management of LU with vulvovaginal candidosis coinfection.

\section{CASE REPORT}

A 20-year-old woman, Batak tribe, an Indonesian citizen, visited the Dermatology and Venereology Department of Sanglah General Hospital in Denpasar with a complaint of ulcer, pain in the pubic area, and vaginal discharge. Initially, the patient experienced a white lumpy stale milk-like vaginal discharge and itching in the pubic area since one month ago. Ulcers in the genital suddenly appeared in 2 weeks without 
being preceded by nodules. The ulcers felt painful, especially during any movement. The patient claimed that she never had any history of sexual intercourse before. She used water and baby soap to wash the genital area without using a female cleanser.

The patient denied any histories of fever, fatigue, or muscle and joint pain. Previously, she was prescribed ciprofloxacin, mefenamic acid, and gentamicin ointment by general practitioners, as well as cefixime and metronidazole from obstetricians. However, the symptoms have not improved. No history of similar symptoms from family. No history of drug and food allergies.

The patient was a student at a state university in Bali. She also joined extracurricular activities and often felt fatigued. History of menarche was at the age of 14 years old, with a history of regular menstruation, and occasional abdominal pain during menstruation. The patient is single, and she never had sexual intercourse, no history of alcohol abuse, wearing contraceptives, or using drugs.

Physical examination showed that the patient was $40 \mathrm{~kg}$ and $148 \mathrm{~cm}$, and she was in a good general condition, and compost mentis level of awareness. Physical examination results of eyes, ears, nose, throat, thorax, abdomen, and extremities were within normal

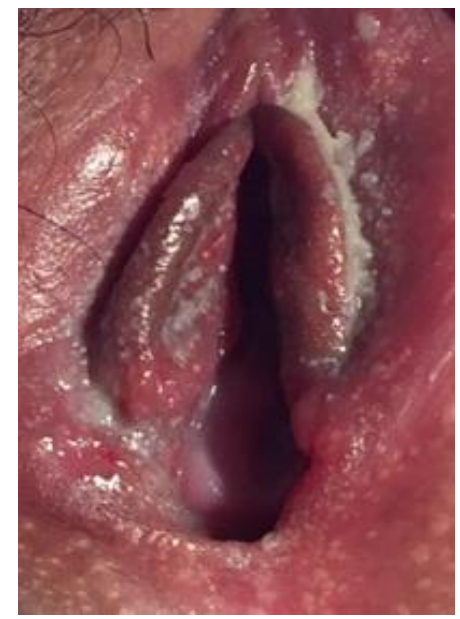

Figure 1. Intact Hymen

Gram examination of the base of the ulcer result showed 1-3 leukocytes per visual field; blastospores $(+)$; Gram-positive cocci (-); coccus basil (-); "school of fish appearance" (-). The amine test result was negative, and the $\mathrm{pH}$ of the vaginal discharge was 4 . The examination of the vaginal discharge showed leukocytes of 20-30 per large visual field; Trichomonas (-), clue cells (-), and 10\% Potassium hydroxide of the vaginal discharge showed blastospores $(+)$ and pseudohifa $(+)$. Gram examination on the vaginal discharge showed 30 leukocytes per limits. There was an enlargement of the left inguinal lymph node, solitary, oval, $1.5 \times 1 \mathrm{~cm}$, with a dense, supple solid consistency. The examination of the nails and mucous oral did not reveal any abnormalities. The examination result of rectal touche showed intact hymen, annular shape (Figure 1). Venereological status in the labia minora (Figure 2) showed efflorescence in the form of ulcers, solitary, geographic shape, $0.6 \times 0.3$ $\mathrm{cm}$, uneven edges, sloping walls, the bottom covered with yellow necrotic tissue, surrounded with skin erythema, soft palpable, and pain. In addition, there were erosions, multiple, geographic shapes, size $0.2 \mathrm{x}$ $0.1 \mathrm{~cm}-0.4 \times 0.2 \mathrm{~cm}$. On the vulva, minimal erythema appears white-colored, vaginal discharged $(+)$, lumpy, and has no odor.

The diagnosis of the patient was established from the history and physical examination. The patient was suspected of having LU with a differential diagnosis of molle ulcer, durum ulcer, and genital herpes ulcers, coinfected with candidiasis vulvovaginal with differential diagnosis of bacterial vaginosis. The supporting examinations were Gram and culture tests. The culture preparation used wet preparations from the base of the ulcer with $10 \%$ Potassium hydroxide, while Gram test from vaginal discharge.

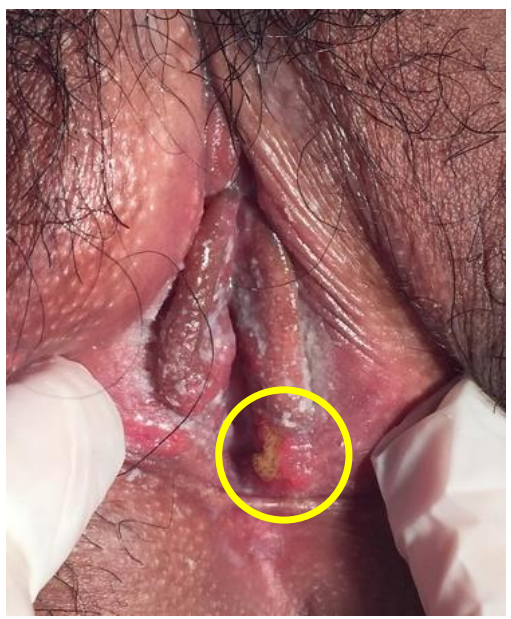

Figure 2. Lipschutz Ulcer.

visual field; blastospores (+); Gram-positive cocci (-); coccus basil (-); diplococcus (-); clue cell (-).

The results of wound culture showed Candida glabrata and sensitive to antifungal fluconazole, voriconazole, caspofungin, micafungin, amphotericin $\mathrm{B}$, and flucytosine. The serological examination results showed IgM and IgG anti-Herpes Simplex Virus (HSV)-1 negative, IgM and IgG anti-HSV-2 negative, EBV VCA negative, IgM and IgG EBV VCA positive. The serological, i.e., Venereal Disease Research Laboratory test (VDRL) and Treponema pallidum 
hemagglutination (TPHA) showed a non-reactive result.

The diagnosis was made based on the history, physical examination, and it was concluded that the patient had LU, co-infected with vulvovaginal candidosis. The patient's management was fluconazole tablets $150 \mathrm{mg}$ single oral dose, mefenamic acid 500 mg orally every 8 hours (prn), and 15-minute wound compress using $\mathrm{NaCl} 0.9 \%$ every $8-12$ hours on the genital ulcer. The patient received IEC (information, education, and communication) regarding the causes and course of the disease, and she was asked to maintain hygiene in the genital area. The observation on day nine showed that vaginal discharge had been reduced, and the genital ulcers dismissed. The venereology status in the vulvar region (Figure 3) showed that erythema was absent with white vaginal discharge $(+)$.

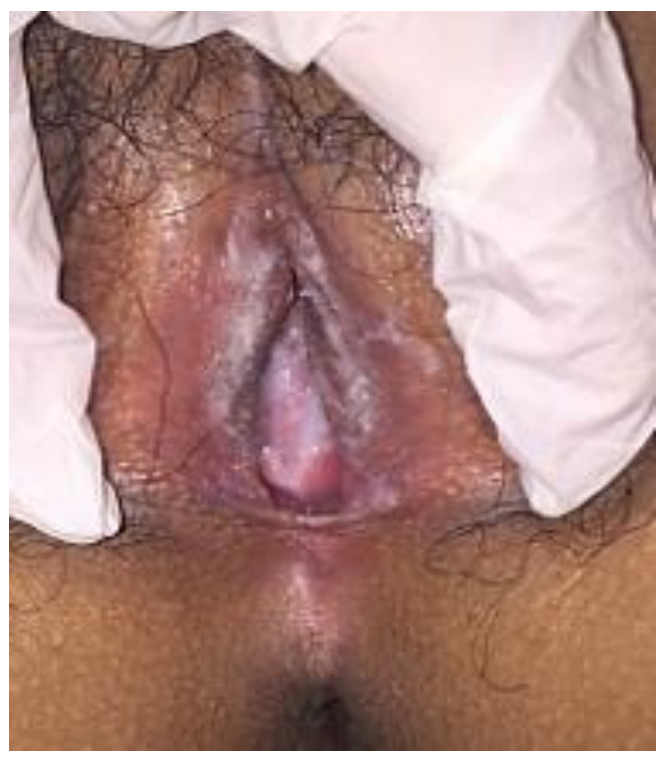

Figure 3. White-colored vaginal discharge.

\section{DISCUSSION}

LU have characteristics of vulva edema and painful necrotic ulcers with rapid onset. The ulcer often affects young non-Sexually active females. ${ }^{1,7,8}$ It is also referred to as an acute non-sexual reactive genital ulcer. Clinical manifestations of LU include symptoms of malaise, flu-like syndrome, fever, fatigue, myalgia, pharyngotonsillitis, lymphadenopathy, and pain accompanied by solitary or multiple ulcers (usually no more than three ulcers), deep and pain in the vulva, especially in the inside part of labia minora. The predilection for the ulcers is inside of the labia and sometimes appears in the labia major, perineum, and lower part of the vagina. The ulcers are soft and painful, well-demarcated, and covered with grayish exudate or pseudomembrane attached; the lesion appears as a purplish ulcer with red margins and a "kissing ulcer" can be found in the vaginal mucosa. Ulcer size varies and can be more than $1 \mathrm{~cm} .{ }^{3,1,2}$

The forms of LU are gangrenous, chronic recurrent, and milliary formis. The gangrenous shape is the most common form, and it has characteristics of acute onset, excruciating, covered with gray-yellowish crusts, surrounded by red margins, especially inside the labia minora and rarely recurrent. Chronic recurrent superficial ulcers are yellowish, small, round, or halfrounded shape with edema. The recurrent predilection of chronic ulcers is in the introitus, labia major, and minor, and usually, heal within 4-6 weeks. Milliary form looks like ulcers with a fibrinous purulent surface, measuring 2-3 mm with inflamed edges, located in the labia major, labia minor, and perineum. The systemic symptoms are mild and able to heal quickly. It is known that the two latter forms may be grouped into the Behcet's disease, and aphthous ulcers, while the gangrenous form is on its kind. ${ }^{5,9}$ In this case, the patient's clinical symptoms in patients suggested gangrenosum form of LU. (*The reasoning of the LU is explained below)

The aetiology of LU is still unknown, but it is often associated with several causes of infection, such as paratyphoid fever, cytomegalovirus infection, Mycoplasma pneumonia, and mostly associated with EBV infection. ${ }^{10,2,5}$ The pathogenesis of LU suggests that EBV may reach the genital mucosa through the hematogenous spread of EBV that infects lymphocytes or the precursors of Langerhans cell. The second mechanism is the theory of autoinoculation in saliva, urine, or cervicovaginal fluid. ${ }^{11}$ Various hypotheses have been proposed relation of the formation of genital 
ulcers and EBV infection, including the formation of immune complexes that causes activation of the tissue complement and necrosis or cytolytic effects of viruscell multiplication between vulvar keratinocyte cells. ${ }^{12}$

An examination for LU can be carried out using laboratory tests such as EBV serological tests and skin biopsy at the lesion border if needed. In this case, EBV serological examination was carried out, and the result of IgM EBV VCA was negative, while the IgG EBV VCA was positive. We hypothesized that the LU lesions were caused by EBV infection that most EBV cases are often associated with LU. In this case, a skin biopsy was not performed because the histopathological examination is not the determinant diagnosis of LU. ${ }^{12}$

One of the LU differential diagnosis is a molle ulcer. This ulcer is caused by Haemophilus ducreyi, a Gram-negative, facultative anaerobic coccobacillus. The ulcer is sexually transmitted with characteristics of multiple acute, painful, soft on palpation, cup-like shaped with irregular edges. The primary form of the granulation tissue that bleeds easily and is covered in gray-yellowish purulent necrotic tissue. ${ }^{13}$ Another differential diagnosis of LU is durum ulcer. Durum ulcers are caused by Treponema pallidum, and they are usually transmitted through sexual contact. Durum ulcer is the primary stage of syphilis, and it is characterized by solitary ocular ulcers or oval-shaped erosions, gentle ramps, clean, the surrounding skin is not inflamed, painless (indolent), and hard on palpation. The usual ulcer sites are the labia minor and labia major. ${ }^{14}$ Genital herpes can also be a differential diagnosis of LU. The cause of genital herpes, in general, is HSV-2 and possibly HSV-1. Its clinical manifestations are multiple vesicles and pustules, which may rupture into erosion or erythema ulcers accompanied by pain such as burning, itching, dysuria, and lymphadenopathy. In women, genital herpes often occurs in the vulva, perineum, buttocks, vagina, or cervix. $^{15}$

The clinical manifestations Lipschutz ulcer is distinguishable from its differential diagnosis. However, the disease requires an additional examination to exclude its differential diagnosis, such as Gram inspection, culture, Dark Field Microscope inspection, serological test (syphilis, HSV and HIV) and Polymerase chain reaction (PCR) for $\mathrm{HSV}$, if needed. ${ }^{2}$ For molle ulcer, a Gram examination may show coccobacillus negative Gram lined up in a row, which is referred to as a school of fish, while in the culture, Haemophilus ducreyi can be found. ${ }^{13}$ Dark Field Microscope (DFM) examination for durum ulcers may show motile spiral-shaped bacteria. ${ }^{14}$ In this case, an additional examination was needed to rule out the differential diagnosis. The Gram examination result showed 1-3 leukocytes per visual field. The patient refused the DFM examination because she felt pain. The serological test results showed that IgM and Ig G anti-HSV-1 were negative; IgM and IgG anti-HSV-2 were negative. The results of the serological test for syphilis, namely VDRL and TPHA were non-reactive. In the culture examination, Candida glabrata was found, and it was sensitive to fluconazole, voriconazole, caspofungin, micafungin, amphotericin $\mathrm{B}$, and flucytosine.

LU diagnosis is mainly based on clinical symptoms. The criteria for LU include 1) the first time having an acute genital ulcer; 2) age $<20$ years; 3 ) no sexual contact in the last three months; 4) immunocompetent; 5) acute onset (sudden emergence and recovery without scarring within six weeks). Minor criteria may appear in 2 types, i.e., 1) one or several painful ulcers, deep, well-demarcated with necrotic and/or fibrinous parts, and 2) ulcers with a characteristic of "kissing ulcer" (ulcers with a mirrorlike distribution of the vulva area). LU diagnosis can be established if it meets five major criteria and at least 1 of the 2 minor criteria. ${ }^{3}$ In this case, the patient had an acute genital ulcer for the first time. The patient was a 20 years old female with no history of sexual intercourse. She was immunocompetent, had acute onset symptoms, and had characteristics of painful ulcers, bordered firmly with necrotic in the center of the ulcer. The patient met the five major criteria and one minor criterion; therefore, a diagnosis of LU was established.

In this case, other than genital wounds, patients also had a complaint of vaginal discharge. Leucorrhoea was observed with itching around the genitals, and it occurred two weeks before the symptom of genital sores. The 10\% Potassium hydroxide examination results of vaginal discharge showed blastospores and pseudohifa. Gram examination result showed 30 leukocytes per visual field and blastospores $(+)$ with a $\mathrm{pH}$ of 4 , as well as a negative amin test.

Vulvovaginal candidiasis (VVC) diagnosis is established based on discharge characteristics and 10\% Potassium hydroxide examination. Differential diagnoses of VVC are all complaints of vaginal discharge, especially bacterial vaginosis or trichomoniasis. ${ }^{16}$ Bacterial vaginosis, which is a clinical manifestation due to changes in normal vaginal flora. It is characterized by gray-whitish vaginal discharge and fishy-smelling odor caused by Gardnerella vaginalis and other anaerobic bacteria such as Bacteroides spp. and Mobiluncus spp. Trichomoniasis is a urogenital tract infection of both females and males caused by Trichomonas vaginalis. 
The clinical symptom of these differential diagnoses is different from VVC, and the diagnosis is confirmed using supporting examination such as Gram and wet preparations. ${ }^{17.18}$ Studies and literature suggested that there is no association between the incidence of VVC and LU. However, other infections, including Candida infections, may present in $\mathrm{LU}^{3}$

LU therapy is mainly symptomatic because the ulcer may heal itself within 16-21 days. Topical therapy includes antibiotics and anesthesia with or without topical corticosteroids. Analgesics with or without broad-spectrum antibiotics or antibiotics based on culture results may be used as systemic therapy. In severe clinical manifestation, such as pain, oral corticosteroids can be given in the short term. ${ }^{1,2,5,9}$

In this LU case management, the patient was prescribed with $\mathrm{NaCl} 0.9 \%$ 15-minute compress every 8 hours to 12 hours on the genital wounds and mefenamic acid $500 \mathrm{mg}$ every 8 hours orally, administered if the pain is present. $\mathrm{NaCl} 0.9 \%$ or other physiological fluid compress is useful for reepithelialization of tissue by providing a moisturizing effect. Mefenamic acid is one of the analgesic drugs from non-steroidal anti-inflammatory class for treating mild to moderate pain.

The therapeutic option for VVC in a non-pregnant woman is miconazole or clotrimazole $200 \mathrm{mg}$ intravaginally, three times a day for three days, or clotrimazole $500 \mathrm{mg}$ intravaginally single dose, or fluconazole $150 \mathrm{mg}$ per single oral dose, or itraconazole $200 \mathrm{mg}$ per single oral dose, or single nystatin dose 100,000 IU intravenously every day for seven days. ${ }^{16}$ In this case, the patient was prescribed fluconazole tablets $150 \mathrm{mg}$ single dose orally, following the antifungal sensitivity test results that give adequate results. An antifungal tablet is also appropriate for women with no history of sexual intercourse. $^{16}$

The clinical manifestation of LU may heal on its own. However, secondary or concomitant infection such as Candida infection is possible. Hygiene plays an essential role in preventing secondary infection and further development of the disease. ${ }^{1.2}$ The prognosis of the patient was dubia ad bonam.

This paper presented a case of LU with vulvovaginal candidiasis in women aged 20 years old who had never been in sexual intercourse. The diagnosis was established based on history, physical examination, and additional examination to exclude other causes of ulcers, especially those caused by sexually transmitted infections. The patient was prescribed with $\mathrm{NaCl} 0.9 \%$ compress for genital wounds, $500 \mathrm{mg}$ mefenamic acid every eight hours orally, and fluconazole $150 \mathrm{mg}$ orally in a single dose, which resulted in clinical improvement. The prognosis of the patient was dubia ad bonam.

\section{REFERENCES}

1. Sehgal VN, Pandhi D, Khurana A. Nonspecific genital ulcers. Clin Dermatol 2014; 32: 259-74.

2. Brinca A, Carvalho MJ, Figueiredo A, Canelas MM, Vieira R. Lipschutz ulcer (ulcus vulvae acutum)-a rare cause of genital lesion. An Bras Dermatol 2012; 87(4): 622-4.

3. Farhi D, Wendling J, Molinari E. Nonsexually related acute genital ulcers in 13 pubertal girls: a clinical and microbiological study. Arch Dermatol 2009; 145: 38-45.

4. Huppert JS. Lipschutz ulcers: evaluation and management of acute genital ulcers in women. Dermatologic Therapy 2010; 23; 533-540.

5. Ueda Y, Kamiya K, Ohtsuki M. Reactive nonsexually related acute genital ulcers. J Gen Fam Med 2018; 19; 30-31.

6. Halvorsen JA, Brevig $\mathrm{T}$, Aas $\mathrm{T}$, Skar AG, Slevolden EM, Moi H. Genital ulcers as initial manifestation of Epstein-Barr virus infection: two new cases and a review of the literature. Acta Derm Venereol 2006; 86: 439-42.

7. Garcia JG. Case report: Lipschutz ulcer. Am J Emerg Med 2016; 12: 26-7.

8. Marcus S, and Neill S. Unusual presentation of acute vulval ulceration. Trend Uro Gyn Sex Health 2008: 24-25.

9. Archel EB, Ruiz Goikoetxea MR, Elizalde ER, Rementería XB, Gómez LG, Lizarraga AI. Lipschütz ulcer in a 17-month-old girl: a rare manifestation of Epstein-Barr primoinfection. Eur J Pediatr 2013; 172(8): 1121-3.

10. Di Lernia V, Mansouri Y. Epstein-Barr Virus and skin manifestations in childhood. Int $\mathrm{J}$ Dermatol 2013; 52: 1177.

11. Sárdy M, Wollenberg A, Niedermeier A, Flaig MJ. Genital ulcers associated with Epstein- Barr virus infection (ulcus vulvae acutum). Acta Derm Venereol 2011; 91(1): 55-9.

12. Barrett MM, Sangueza M, Werner B, et al. Lymphocytic arteritis in Epstein-Barr Virus vulvar ulceration (Lipschutz disease): A report of 7 cases. Am J Dermatopathol 2015;37(9);691-8.

13. Lautenschlager S. Chancroid. In: Goldsmith LA, Katz SI, Gilchrest BA, Paller AS, Leffell DJ, Wolff K, editors. Fitzpatrick's Dermatology in General Medicine, $8^{\text {th }}$ edition. New York: McGraw-Hill 2012. p. 2501-4.

14. Katz KA. Syphilis. In: Goldsmith LA, Katz SI, Gilchrest BA, Paller AS, Leffell DJ, Wolff K, editors. Fitzpatrick's Dermatology in General 
Medicine, $8^{\text {th }}$ edition. New York: McGraw-Hill 2012. p. 2471-93.

15. Marques AR, and Cohen JI. Herpes simplex. In: Goldsmith LA, Katz SI, Gilchrest BA, Paller AS, Leffell DJ, Wolff K, editors. Fitzpatrick's Dermatology in General Medicine, $8^{\text {th }}$ edition. New York: McGraw-Hill 2012. p. 2367-82.
16. Paladine HL. Vaginitis diagnosis and treatment. Am Fam Physician 2018; 97(5);321-329.

17. Schalkwyk JV. Vulvovaginitis: Screening for and management of trichomoniasis, vulvovaginal candidiasis, and bacterial vaginosis. J Obstet Gynaecol Can 2015; 37(3): 266-274.

18. Kissinger P. Trichomonas vaginalis: a review of epidemiologic, clinic and treatment issues. BMC Infectious Disease 2015; 15: 307. 